\title{
DUAL SPACES OF A VECTOR LATTICE AND ITS CUT-COMPLETION
}

\author{
BY \\ J. J. MASTERSON
}

Introduction. Let $L$ be an Archimedean vector lattice. The space $L_{n}^{\tilde{n}}$ of all normal integrals (order continuous linear functionals) on $L$ was first studied by H. Nakano [6], and has been the object of recent investigation [1]-[5].

The central theorem in this paper shows that if $\hat{L}$ is the cut-completion of $L$, then $L_{n}^{\tilde{n}}$ and $(\hat{L})_{n}^{\tilde{n}}$ are isomorphic vector lattices, a known result when $L=C(X)$, the continuous functions on a compact Hausdorff space (cf. [3, §10]). This serves as a convenient tool for examining the role that completeness of $L$ plays in determining properties of $L_{\tilde{n}}$. It will be shown that a number of results previously obtained under the assumption that $L$ is complete can be extended to Archimedean vector lattices.

1. The isomorphism of $L_{n}^{\tilde{n}}$ and $(\hat{L})_{n}^{\tilde{n}}$. We first recall some definitions and other facts basic to our study of the cut-completion (or Dedekind completion) of a vector lattice. We will say that a subvector lattice $D$ of $L$ is order dense in $\hat{L}$ (or dense in $L$ ) if for every positive element $a$ in $L, a=\inf (B)$ for some set $B$ of positive elements taken from $D . \hat{L}$ then is said to be a cut-completion of the vector lattice $L$ if $\hat{L}$ is a complete vector lattice which contains a dense subvector lattice isomorphic to $L$ ("isomorphism" always denotes vector lattice isomorphism). To simplify notation we will consider $L$ to be a subvector lattice of $\hat{L}$.

The following three properties of $\hat{L}$ are well known and will be used in the paper without proof:

(a) $\hat{L}$ is unique up to isomorphism.

(b) If $B$ is an arbitrary subset of elements of $L$ and the supremum of $B$ taken in $L$ exists, then it is identical with the supremum of $B$ taken in $\hat{L}$.

(c) If $\hat{a}$ is a positive element of $\hat{L}$, then there exist subsets $B$ and $C$ of $L$ such that $\hat{a}=\sup (B)=\inf (C)$.

Let $L$ be an Archimedean vector lattice and $\hat{L}$ its cut-completion. Then,

THEOREM 1.1. $L_{n}^{\tilde{n}}$ and $(\hat{L}) \tilde{n}$ are isomorphic rector lattices $\left({ }^{1}\right)$.

Proof. Let $f \geqq 0$ be a normal integral on $L$ and extend it to the positive cone $\hat{L}^{+}$ of $\hat{L}$ by

$$
\hat{f}(\hat{a})=\sup \{f(x): 0 \leqq x \leqq \hat{a}, x \in L\}, \quad \hat{a} \in \hat{L}
$$

Received by the editors March 1, 1966 and, in revised form, October 26, 1966.

( $\left.{ }^{1}\right)$ The author wishes to thank the referee for simplifying the proof of Theorem 1.1. 
The supremum of a set in $L$ exists and agrees with that in $\hat{L}$ from which it follows that $\left.\hat{f}\right|_{L^{+}}=f$. Moreover, $\hat{f}$ is monotone on $\hat{L}^{+}$.

(a) $\hat{f}(\hat{a})=\inf \{f(y): \hat{a} \leqq y \in L\}$. Clearly, $\hat{f}(\hat{a}) \leqq \inf \{f(y): \hat{a} \leqq y \in L\}$. Suppose there is an $\varepsilon>0$ such that $\hat{f}(\hat{a})+\varepsilon \leqq \inf \{f(y): \hat{a} \leqq y \in L\}$. Then,

$$
\inf _{\hat{L}}\{y-x: 0 \leqq x \leqq \hat{a} \leqq y ; x, y \text { in } L\}=0=\inf _{L}\{y-x: 0 \leqq x \leqq \hat{a} \leqq y ; x, y \text { in } L\} .
$$

But $f$ would not then be order continuous, contradicting the fact that it is a normal integral.

(b) If $B \subset \hat{L}^{+}, \hat{f}(\inf B)=\inf (\hat{f}(B))$. Again, $\hat{f}(\inf B) \leqq \inf (\hat{f}(B))$ is clear. Let $\varepsilon>0$ and inf $B=\hat{b}_{0}$. There exists $x \in L, x \geqq \hat{b}_{0}$ with $\hat{f}\left(\hat{b}_{0}\right)+\varepsilon \geqq f(x)$. Then by the monotonicity of $f$ there is $\hat{b} \in B$ with $\hat{b} \leqq x$, whence $\hat{f}\left(\hat{b}_{0}\right)+\varepsilon \geqq \hat{f}(\hat{b}) \geqq \inf (\hat{f}(B)) \hat{f}$, then, is order continuous on $\mathcal{L}$.

(c) $\hat{f}$ is additive on $\hat{L}^{+}$. Let $\hat{a}_{1}$ and $\hat{a}_{2}$ be in $\hat{L}$.

$$
\left\{\left[0, \hat{a}_{1}\right] \cap L\right\} \cup\left\{\left[0, \hat{a}_{2}\right] \cap L\right\} \subset\left[0, \hat{a}_{1}+\hat{a}_{2}\right] \cap L .
$$

So,

$$
\begin{aligned}
\hat{f}\left(\hat{a}_{1}\right)+\hat{f}\left(\hat{a}_{2}\right) & =\sup \left(f\left(\left[0, \hat{a}_{1}\right] \cap L\right)\right)+\sup \left(f\left(\left[0, \hat{a}_{2}\right] \cap L\right)\right) \\
& =\sup \left(f\left(\left\{\left[0, \hat{a}_{1}\right] \cap L\right\} \cup\left\{\left[0, \hat{a}_{2}\right] \cap L\right\}\right)\right) \\
& \leqq \sup \left(f\left(\left[0, \hat{a}_{1}+\hat{a}_{2}\right] \cap L\right)\right) \\
& =\hat{f}\left(\hat{a}_{1}+\hat{a}_{2}\right) .
\end{aligned}
$$

Let $\varepsilon>0$. Then, there exists $b \in\left[0, \hat{a}_{1}+\hat{a}_{2}\right] \cap L$ with $\hat{f}\left(\hat{a}_{1}+\hat{a}_{2}\right)-\varepsilon \leqq f(b)$. Let $b=\hat{b}_{1}+\hat{b}_{2}$. Then, $\hat{f}\left(\hat{b}_{2}\right)=\hat{f}\left(b-\hat{b}_{1}\right)=f\left(\inf \left\{b-x: 0 \leqq x \leqq \hat{b}_{1}, x \in E\right\}\right)=f(b)-\hat{f}\left(\hat{b}_{1}\right)$ applying the decomposition lemma for vector lattices. So, $\hat{f}\left(\hat{a}_{1}+\hat{a}_{2}\right)-\varepsilon \leqq f(b)=\hat{f}\left(\hat{b}_{1}\right)+\hat{f}\left(\hat{b}_{2}\right)$ $\leqq \hat{f}\left(\hat{a}_{1}\right)+\hat{f}\left(\hat{a}_{2}\right)$. Equality then follows.

(d) Positive homogeneity is clear. Setting $\hat{f}(\hat{x})=\hat{f}\left(\hat{x}^{+}\right)-\hat{f}\left(\hat{x}^{-}\right)$for arbitrary $\hat{x} \in \hat{L}$, then, $\hat{f}$ becomes a normal integral on $\hat{L}$. $\hat{f}$ is uniquely determined by $f$ and it follows rather easily that the mapping $f \rightarrow \hat{f}$ is the desired isomorphism.

If $L_{n}^{\tilde{n}}$ is separating on $L$, then $L$ can be embedded in $L_{n}^{\sim} \sim\left(L_{n}^{\tilde{n}} \sim=\left(L_{n}^{\tilde{n}}\right)_{n}^{\tilde{n}}\right)$ as a subvector lattice. If $L$ is complete, then under this embedding it is an ideal in $L_{n}^{\sim} \sim$ (cf. $[1,3.8])$. So, $\hat{L}$ is an ideal in $(\hat{L})_{n}^{\sim} \sim$. With the above theorem, this gives us the following:

COROLLARY 1.2. $L$ can be embedded in $L_{n}^{\sim} \sim$ as an ideal.

Moreover, it is easily seen that $\hat{L}$ is the ideal in $L_{n}^{\sim} \sim$ generated by $L$, a somewhat surprising relation between the concepts of cut-completion and continuous linear functional.

We will now apply the theorem to obtain a result concerning absolute weak topologies on $L_{n}^{\tilde{n}}$. The absolute weak topology on $L_{n}^{\tilde{n}}$ generated by $L$ is the locallyconvex topology defined in $L_{n}^{\tilde{n}}$ by the polars of the bounded sets in $L$. Equivalently, if for each positive element $a$ in $L$, we define a seminorm \|\|$_{a}$ on $L_{n}^{\tilde{n}}$ by 
$\|f\|_{a}=|f|(a)$, the absolute weak topology is the topology defined by the family of all such seminorms. We will denote this topology by $|w|\left(L_{n}^{\tilde{n}}, L\right)$.

Using the seminorm definition of the topology and the fact that for every positive element $\hat{a}$ in $\hat{L}$ there is a positive element $a$ in $L$ such that $a \geqq \hat{a}$, and for every positive element $b$ in $L$ there is a positive element $\hat{b}$ in $\hat{L}$ such that $\hat{b} \geqq b$, we obtain the following:

COROLlaRY 1.3. The $|w|\left(L_{\tilde{n}}^{\tilde{n}}, L\right)$ and $|w|\left(L_{\tilde{n}}, \hat{L}\right)$ topologies on $L_{\tilde{n}}^{\tilde{n}}$ are identical.

The following two results remove the hypothesis that $L$ be a complete vector lattice from theorems appearing in [2] and [3]; ([3, 2.8] and [2, 3.3] respectively).

COROLlaRY 1.4. If $I$ is a dense ideal in $L$, then $L_{\tilde{n}}^{\tilde{c}}$ can be embedded in $I_{n}^{\tilde{n}}$ as a dense ideal.

Proof. The cut-completion of $I$ has a natural embedding in $\hat{L}$ as a dense ideal. We call this ideal $\tilde{I}$. By Theorem 1.1, $I_{n}^{\sim} \cong(\hat{I})_{n}^{\sim}$ and $L_{n}^{\tilde{n}} \cong(\hat{L})_{n}^{\tilde{n}}$. Applying the analogous theorem in [3] to $\hat{I}$ and $\hat{L}$, the result follows.

A subvector lattice $D$ of $L$ is said to be closed in $L$ if whenever $B \subset D$ is a set of positive elements such that $\sup (B)$ is in $L$, then $\sup (B)$ is in $D$.

CoROllary 1.5. If I is a closed ideal in $L_{\tilde{n}}^{\tilde{n}}$, then $I$ is $w\left(L_{\tilde{n}}^{\tilde{n}}, L\right)$ closed.

Proof. (The proof is a variation of that in [2], letting $J$ be the null ideal of $\phi$ in $\hat{L}$ instead of $L$.) $L_{n}^{\tilde{n}}=I \oplus I^{\prime}$ since $I$ is closed. It suffices to show that if $\phi \in I^{\prime}$ and $\phi \neq 0$, then $\phi$ is not a $w\left(L_{n}^{\tilde{n}}, L\right)$ limit point of $I$. Now, the null ideal $J$ of $\phi$ in $\hat{L}$ is closed ([2], 3.3) hence $\hat{L}=J \oplus J^{\prime}$. So, $L_{n}^{\tilde{n}}=J^{\perp} \oplus J^{\prime \perp}$. Let us first show that $J^{\prime \perp}$ is $w\left(L_{n}^{\tilde{n}}, L\right)$ closed. Let $\left\{f_{\alpha}\right\} \subset J^{\prime \perp}$ and $f \in L_{n}^{\tilde{n}}$ be such that $f_{\alpha} \rightarrow f\left(w\left(L_{n}^{\tilde{n}}, L\right)\right)$ i.e., $f_{\alpha}(a) \rightarrow f(a)$ for all $a \in L$. But then for $a \in J^{\prime} \cap L, f_{\alpha}(a)=0$ for all $\alpha$, since $f_{\alpha} \in J^{\prime \perp}$. So, $f(a)=0$ for each $a \in J^{\prime} \cap E$. Hence for $\hat{a} \in J^{\prime}, f(\hat{a})=0$ ( $f$ being continuous and $L$ being order dense in $\hat{L}$.) So, $f \in J^{\prime \perp}$ and hence $J^{\prime \perp}$ is $w\left(L_{\tilde{n}}, L\right)$ closed. Now $\phi \in J^{\perp}$ hence not in $J^{\prime \perp}$. So, we can show that $\phi$ is not a weak limit point of $I$ by showing that $I \subset J^{\perp \perp}\left(J^{\prime \perp}\right.$ being weakly closed). Since $L_{n}^{\tilde{n}}=J^{\perp} \oplus J^{\perp}$ we establish $I \subset J^{\perp}$ by proving that $I \cap J^{\perp}=\{0\}$.

Consider any $\psi \in I, \psi>0$. We have to show $\psi(a) \neq 0$ for some $a \in J$. Now, $\psi \wedge|\phi|=0$. So, for $\varepsilon>0$ and $b \in \hat{L}^{+}$, there is $c$ in $\hat{L}$ such that $0 \leqq c \leqq b$ and $|\phi|(c)+\psi(b-c) \leqq \varepsilon$. It follows easily that there is a sequence $c_{1} \geqq c_{2} \geqq \cdots \geqq 0$ in $\hat{L}$ satisfying $|\phi|\left(c_{n}\right) \leqq 1 / 2^{n}$ and $\psi\left(c_{n}\right) \geqq \lambda>0$ for all $n$. Let $a=\bigwedge_{n} c_{n}$. Then, $|\phi|(a)=0$ which implies that $a \in J$ and since $\psi$ is continuous, $\psi(a)>0$.

REMARK. The proof of 1.5 would be trivial if the topologies $w\left(L_{n}^{\tilde{n}}, L\right)$ and $w\left(L_{\tilde{n}}, \hat{L}\right)$ were identical, as is the case with the $|w|$-topologies. This is not true, however, as the following example shows: Let $L=c$ (convergent sequences). Then $L_{n}^{\tilde{n}}=l^{1}$ and $\hat{L}=l^{\infty}$. Define $x_{n}=\left(x_{n}^{(1)}, x_{n}^{(2)}, \ldots\right)$ by: $x_{n}^{(k)}=0, k \neq n, n+1, x_{n}^{(n)}=1, x_{n}^{(n+1)}$ $=-1$. Then $\left\{x_{n}\right\}$ is a sequence in $l^{1}$ which converges to 0 in the $w\left(l^{1}, c\right)$ topology but not in the $w\left(l^{1}, l^{\infty}\right)$ topology. 
2. The space of normal integrals. In [5], Zaanen and Luxemburg extend a number of the results of [4] from complete to Archimedean vector lattices. New (and often simpler) proofs of these results will be given based on Theorem 1.1 and the elementary propositions listed below. Moreover, natural extensions to the Archimedean case are obtained for several other theorems in [4].

We will first recall some definitions from [4] and state some elementary facts that we need.

If $\phi \in L_{n}^{\tilde{n}}$, then $N_{\phi}=\{a \in L:|\phi||a|=0\}$ is called the null ideal of $\phi$ in $L$. We denote the null ideal of $\phi$ in $\hat{L}$ by $\hat{N}_{\phi}$. The carrier of $\phi$ in $L$ is the complement of $N_{\phi}$ in $L$. I.e., $C_{\phi}=\left(N_{\phi}\right)^{p}=\left\{b \in L:|a| \wedge|b|=0\right.$ for all $\left.a \in N_{\phi}\right\}$. $\hat{C}_{\phi}$ is the carrier of $\phi$ in $\mathcal{L}$. If $A \subset L$, the annihilator of $A$ in $L_{n}^{\tilde{n}}$ is the set $\left\{\phi \in L_{n}^{\tilde{n}}: \phi(a)=0\right.$ for all $\left.a \in A\right\}$ and will be denoted by $A^{\#}$. If $B \subset L_{n}^{\tilde{n}},{ }^{0} B=\{a \in L: \phi(a)=0$ for all $\phi \in B$ is called the annihilator of $B$ in $L .{ }^{\circ} B$ denotes the annihilator of $B$ in $\hat{L}$.

Theorem 1.1 tells us, then, that a normal integral on $L$ may be regarded as a normal integral on $\mathcal{L}$ and vice versa. We will use this fact without mention in proving the following propositions.

From the definition of $N_{\phi}$ and the above-mentioned fact we obtain immediately

Proposition 2.1. If $\phi \in L_{\tilde{n}}^{\tilde{n}}$, then $N_{\phi}=\hat{N}_{\phi} \cap L$.

Proposition 2.2. If $\mathcal{I}$ is an ideal in $\hat{L}$ and $I=I \cap L$, then $(\hat{I})^{p} \cap L=I^{p}$.

Proof. $I$ is dense in $\mathcal{I}$. Then, if $a \in I^{p},|a| \wedge|b|=0$ for all $b \in I$. Given $\hat{b} \in \mathcal{I}$, there is a net $\left\{b_{\alpha}\right\} \subset I$ such that $\left|b_{\alpha}\right| \uparrow|\hat{b}|$. But $|a| \wedge\left|b_{\alpha}\right|=0$ for all $\alpha$. Hence $|a| \wedge|\hat{b}|=0$ which implies that $a \in(\hat{I})^{p} \cap L$, hence $I^{p} \subset(\hat{I})^{p} \cap L$.

Let $a \in(\hat{I})^{p} \cap L$. Then $|a| \wedge|\hat{b}|=0$ for all $\hat{b} \in \hat{I}$ implies $|a| \wedge|b|=0$ for all $b \in I$ since $I \subset I$. So, $a \in I^{p}$ and hence $(\hat{I})^{p} \cap L \subset I^{p}$. Thus, $(\hat{I})^{p} \cap L=I^{p}$.

Since $C_{\phi}=\left(N_{\phi}\right)^{p}$ by definition, the following is an immediate consequence of 2.1 and 2.2 .

Corollary 2.3. If $\phi \in L_{n}^{\tilde{n}}, C_{\phi}=\hat{C}_{\phi} \cap L$.

Proposition 2.4. If $I$ is an ideal in $L$ and $I=\hat{I} \cap L$, then for $\phi \in L_{n}^{\tilde{n}}, \phi(I)=0$ if and only if $\phi(\hat{I})=0$, i.e., $(\hat{I})^{\#}=I^{\#}$.

Proof. Since $I \subset I, \phi(\hat{I})=0$ implies $\phi(I)=0$. Suppose $\phi(I)=0$. Then, if $\hat{a} \in \hat{I}^{+}$, there is a net $\left\{a_{\alpha}\right\}$ in $I$ such that $a_{\alpha} \uparrow \hat{a}$. Since $\phi$ is continuous $\phi\left(a_{\alpha}\right) \rightarrow \phi(a)$. Hence $\phi(\hat{a})=0$. So, $\phi(\hat{I})=0$.

By definition, ${ }^{\circ} B$ is precisely those elements of ${ }^{\hat{0}} B$ which lie in $L$. We state more precisely

Proposition 2.5. If $B$ is a subset of $L_{n}^{\tilde{n}}$, then $\left({ }^{\hat{0}} B\right) \cap L={ }^{0} B$.

Proposition 2.6. If $\hat{L}=\hat{I} \oplus \hat{J}, I=\hat{I} \cap L$ and $J=\hat{J} \cap L$, then $L=\{I \oplus J\}$.

Proof. By [5, Theorem 29.10], $L=\left\{I \oplus I^{p}\right\}$. Since $\hat{L}=\hat{I} \oplus \hat{J}, \hat{J}=(\hat{I})^{p}$. Hence $J=\hat{J} \cap L=(\hat{I})^{p} \cap L=I^{p}$ by Proposition 2.2. So $L=\{I \oplus J\}$. 
The following theorems are proved in [5]. Proofs will be given here based on Theorem 1.1 and the results for Dedekind complete vector lattices in [4]. We denote the set of normal integrals on both $L$ and $\hat{L}$ by $L_{n}^{\tilde{n}}$.

THEOREM 2.8. If $L$ is Archimedean and if $\phi, \Psi \in L_{n}^{\tilde{n}}$, then the following conditions are mutually equicalent: (a) $|\phi| \wedge|\Psi|=0$, (b) $C_{\phi} \subset N_{\Psi}$, (c) $C_{\Psi} \subset N_{\phi}$, (d) $C_{\phi} \perp C_{\Psi}$ (cf. $[5,31.2])$.

Proof. By [4, Theorem 27.6], the following are equivalent: (a') $|\phi| \wedge|\psi|=0$, (b') $\hat{C}_{\phi} \subset \hat{N}_{\psi},\left(c^{\prime}\right) \hat{C}_{\psi} \subset \hat{N}_{\phi}$, (d') $\hat{C}_{\phi} \perp \hat{C}_{\psi}$. To complete the argument, it suffices to show that (b) is equivalent to $\left(\mathrm{b}^{\prime}\right)$ and $(\mathrm{d})$ is equivalent to $\left(\mathrm{d}^{\prime}\right)$. By 2.1 and 2.3, (b') implies (b). Assume (b) i.e., $C_{\phi} \subset N_{\psi}$. Let $a \in \hat{C}^{+}$. Then, there is a net $\left\{a_{\alpha}\right\} \subset C_{\phi}$ such that $a_{\alpha} \uparrow a$. But then $\left\{a_{\alpha}\right\} \subset N_{\psi}$. So $|\psi|\left(\left|a_{\alpha}\right|\right)=0$ for all $\alpha$ and hence $|\psi|(|a|)=0$. Thus, $a \in \hat{N}_{\psi}$ hence $\hat{C}_{\phi} \subset \hat{N}_{\psi}$. Using the density of $C_{\phi}$ in $\hat{C}_{\phi}$ and 2.3 , the equivalence of (d) and ( $\left.d^{\prime}\right)$ follows easily.

Corollary 2.9 (Hahn Decomposition). Let L be Archimedean. Given $\phi \in L_{n}^{\tilde{n}}$, there exist normal subspaces $P$ and $N$ such that $P \cap N=\{0\}, P \oplus N$ is (order) dense in $L, \phi(u) \leqq 0$ for all $0 \leqq u \in N$ and $\phi(u) \geqq 0$, for $0 \leqq u \in P$. Moreover, $P=C_{\phi^{+}}$and $N=N_{\phi^{+}}(c f .[5,31.3])$.

Proof. By [4, Corollary 27.7], there exist $\hat{P}$ and $\hat{N}$ such that $\hat{L}=\hat{P} \oplus \hat{N}(\hat{P}$ and $\hat{N}$ are normal subspaces) such that $\phi(u) \geqq 0$ for all $0 \leqq u \in \hat{P}$ and $\phi(u) \leqq 0$ for all $0 \leqq u \in \hat{N}$. Moreover, $\hat{P}=\hat{C}_{\phi^{+}}$and $\hat{N}=\hat{N}_{\phi^{+}}$. Let $P=\hat{P} \cap L$ and $N=\hat{N} \cap L$. Then, by 2.1 and 2.3, $N=N_{\phi^{+}}$and $P=C_{\phi^{+}} . \hat{P} \cap \hat{N}=\{0\}$ hence $P \cap N=\{0\}$. Also, since $P \subset \hat{P}$ and $N \subset \hat{N}, \phi(u) \leqq 0,0 \leqq u \in N$ and $\phi(u) \geqq 0,0 \leqq u \in P$. By $2.6,\{P \oplus N\}=L$. Since $\hat{P}$ is a normal subspace of $\hat{L}, P=\hat{P} \cap L$ is a normal subspace of $L$ and likewise for $N$. This completes the argument.

Theorem 31.5 in [5] can be generalized without using the lemma which precedes it.

TheOREM 2.10. Let $L$ be Archimedean. If $B$ is an ideal in $L_{n}^{\tilde{n}}$, then $\left({ }^{\circ} B\right)^{\#}=\{B\}$.

Proof. Since $\{B\}$ is a normal subspace, it suffices to consider $B$ to be a normal subspace of $L_{n}^{\tilde{n}}$. Then, by [4, Theorem 27.10], $\left({ }^{\hat{0}} B\right)^{\#}=B=\{B\}$. But $\left({ }^{0} B\right)^{\#}=\left({ }^{\circ} B \cap L\right)^{\#}$ $=\left({ }^{\hat{O}} B\right)^{\#}$ by 2.5 and 2.4. Applying $\left[4\right.$, Theorem 27.10], we have $\left({ }^{\circ} B\right)^{\#}=\left({ }^{\hat{0}} B\right)^{\#}=\{B\}$.

If $B$ is an ideal in $L_{n}^{\tilde{n}}$, then the normal subspace of $L$ generated by all carriers $C_{\phi}$, where $\phi \in B$, is called the carrier of $B$ and is denoted by $C_{B}$.

Let $\hat{C}_{B}$ denote the carrier of $B$ in $\hat{L}$. Then, we note that $\hat{C}_{B} \cap L=C_{B}$. Clearly, $C_{B} \subset \hat{C}_{B} \cap L$ since for each $\phi \in B, C_{\phi} \subset \hat{C}_{\phi} \cap L$. Now suppose $a \in \hat{C}_{B} \cap L$ is a positive element. Then, there is a net $\left\{\hat{a}_{\alpha}\right\}$ such that for each $\alpha, \hat{a}_{\alpha} \in \hat{C}_{\phi}$ for some $\phi \in B$, and such that $\hat{a}_{\alpha} \uparrow a$. For each $\alpha$, there is a net $\left\{a_{\alpha, \beta}\right\} \subset L$ such that $a_{\alpha, \beta} \uparrow \hat{a}_{\alpha}$. Then, $a=\bigvee a_{\alpha, \beta}-i n-\hat{L}$ and hence $a=\vee a_{\alpha, \beta}-i n-L$. But $a_{\alpha, \beta} \in C_{\phi}$ for some $\phi$, given $\alpha$ and $\beta$. Hence $a \in C_{B}$. 
Theorem 2.11. (i) If $L$ is Archimedean and $B$ is an ideal in $L_{n}^{\tilde{n}}$, then $C_{B}=\left({ }^{0} B\right)^{p}$. Hence, $L=\left\{{ }^{0} B \oplus C_{B}\right\}$. In addition, the ideals $B$ and $B_{1}$ in $L_{n}^{\tilde{n}}$ are disjoint if and only if $C_{B}$ and $C_{B_{1}}$ are disjoint.

(ii) If $L$ is Archimedean and $B$ is an ideal in $L_{n}^{\tilde{n}}$, then $C_{B}=C_{\{B\}}(c f .[5,31.8])$.

Proof. By [4, Theorem 27.12], $\hat{C}_{B}=\left({ }^{\hat{0}} B\right)^{p}$. By the remark made above, $C_{B}=\hat{C}_{B} \cap L$. But then, $C_{B}=\hat{C}_{B} \cap L=\left({ }^{0} B\right)^{p} \cap L=\left({ }^{0} B\right)^{p}$ by 2.2 and 2.5. Using again the remark made above, it is easily shown that $C_{B} \perp C_{B_{1}}$ if and only if $\hat{C}_{B} \perp \hat{C}_{B_{1}}$. But it is proved in [4, Theorem 27.12] that ideals $B$ and $B_{1}$ in $L_{n}^{\tilde{n}}$ are disjoint if and only if $\hat{C}_{B_{1}}$ and $\hat{C}_{B}$ are disjoint. The last statement in (i) then follows.

Since $C_{B}=\hat{C}_{B} \cap L$, and from [4, Theorem 27.12], $\hat{C}_{B}=\hat{C}_{(B)}$, (ii) is proved.

$L_{\tilde{n}}^{\tilde{n}}$ is a normal subspace in $L^{\sim}$. Then, let $L_{0}={ }^{0}\left(L_{n}^{\tilde{n}}\right)$ and let $L_{1}$ be the carrier of $L_{n}^{\tilde{n}}$ in $L$. Then, $L_{1}=\left(L_{0}\right)^{p}$ and $L=\left\{L_{0} \oplus L_{1}\right\}$.

THEOREM 2.12. (i) If $L$ is Archimedean and $A$ is a normal subspace of $L_{1}$, then ${ }^{\circ}\left(A^{\#}\right)=\left\{A \oplus L_{0}\right\}$. (ii) If $L$ is Archimedean and $A$ is a normal subspace of $L_{1}$, then $A$ is the carrier of some normal subspace of $L_{n}^{\tilde{n}}$ (cf. [5, Theorem 31.10]).

Proof. (i) If $A$ is a normal subspace of $L_{1}$, then $\hat{A}=\{b \in \hat{L}:|b| \leqq a$ for some $a \in L\}$ is a normal subspace of $\hat{L}_{1}$. So, by [4, Theorem 27.14], ${ }^{\circ}\left(\hat{A}^{\#}\right)=\hat{A} \oplus \hat{L}_{0}$. Now ${ }^{\circ}\left(\hat{A}^{\#}\right) \cap L={ }^{\circ}\left(A^{\#}\right)$ by 2.5 and 2.4. By 2.6, $\left(\hat{A} \oplus \hat{L}_{0}\right) \cap L=\left\{A \oplus L_{0}\right\}$; hence (i) follows.

(ii) If $A$ is a normal subspace of $L_{1}$, then $A$ is a normal subspace of $L_{1}$. But then, $\hat{A}$ is by [4, Theorem 27.14], the carrier of some normal subspace $\hat{B}$ of $L_{\tilde{n}}$. So, by 2.2 and 2.5 , and the fact that $A=\hat{A} \cap L, A$ is the carrier of $\hat{B}$ in $L$.

It is also possible, using 1.1 , to prove Archimedean analogs of other results proved in [4] for Dedekind complete vector lattices.

Recall that a vector lattice $L$ has the Egoroff property in case $0 \leqq u_{n, k} \uparrow u$, $n=1,2, \ldots$ implies the existence of a sequence $0 \leqq v_{m} \uparrow u$ such that for every pair of indices $(m, n)$ there exists $j=j(m, n)$ satisfying $v_{m} \leqq u_{n j}$. The concept "superDedekind complete" can be extended in a natural way to an Archimedean vector lattice $L$ by omitting the condition that $L$ be Dedekind complete: An Archimedean vector lattice is called super-Archimedean in case $0 \leqq u_{\tau}$ and $u_{\tau} \uparrow u_{0}$ implies the existence of a subsequence $\left\{u_{\tau_{n}}\right\}$ of $u_{\tau}$ such that $u_{\imath_{n}} \uparrow u_{0}$. Then [4, Theorem 27.17] can be extended using the following lemma.

LEMmA 2.13. If $\hat{I} \subset \hat{L}$ is an ideal and $I$ is super-Dedekind complete and has the Egoroff property, then $I=I \cap L$ is super-Archimedean and has the Egoroff property.

Proof. $I$ is clearly super-Archimedean since it inherits this property from $\hat{I}$. Suppose $0 \leqq u_{n, k} \uparrow u$ for each $n=1,2, \ldots ; u \in I, u_{n, k} \in I, n, k=1,2, \ldots$ Then, there exists a sequence $\left\{\hat{v}_{m}\right\} \subset \hat{I}$ such that $\hat{v}_{m} \uparrow u$ and such that for every pair of indices $m, n$, there exists $j=j(m, n)$ such that $\hat{v}_{m} \leqq u_{n, j}$. Now, there is a net $\left\{v_{m, \tau}\right\} \subset I$ such that $0 \leqq v_{m, \tau} \uparrow \hat{v}_{m}$ for each $m$ and hence, since $\hat{I}$ is super-Dedekind complete, a sequence $\left\{v_{m, k}\right\} \subset I$ such that $v_{m, k} \uparrow \hat{v}_{m}$ for each $m$. Extend the countable set $\left\{v_{m, k}, k=1,2, \ldots\right.$; $m=1,2, \ldots\}$ to a countable directed set $\left\{w_{v}\right\}$ by adding all suprema of finite 
subsets of $\left\{v_{m, k}\right\} .\left\{w_{v}\right\}$ is then a sequence of elements of $I$ such that $w_{v} \uparrow u$ (since $v_{m, k} \uparrow \hat{v}_{m}$ for each $m$ and $\hat{v}_{m} \uparrow u$ ). Moreover, given $\nu, w_{v}=v_{m_{1}, k_{1}} \vee \cdots \vee v_{m_{p}, k_{p}}$ and $v_{m_{1}, k_{1}} \leqq \hat{v}_{m_{1}}, \ldots, v_{m_{p}, k_{p}} \leqq \hat{v}_{m_{p}}$. But then, since $\left\{\hat{v}_{m}\right\}$ is directed there is an $m^{\prime}$ such that $\hat{v}_{m_{1}} \vee \cdots \vee \hat{v}_{m_{p}} \leqq \hat{v}_{m^{\prime}}$ so, $w_{v} \leqq \hat{v}_{m^{\prime}}$. Then, if $\nu$ and $n$ are given, there is $j$, namely $j=j\left(m^{\prime}, n\right)$ such that $\hat{v}_{m^{\prime}} \leqq u_{n, j}$ and hence $w_{v} \leqq u_{n, j}$. So, $I$ has the Egoroff property.

We recall from [4] the definition of Riesz direct sum. $A \subset L$ will be called the Riesz direct sum of the collection of normal subspaces $\left\{A_{\tau}\right\}$ if all $A_{\tau}$ are mutually disjoint and if $A$ is the smallest normal subspace containing all $A_{\tau}$. We use the notation $A=\bigcup \oplus A_{\tau}$.

Lemma 2.14. If $\hat{A} \subset \hat{L}$ and $\hat{A}=\bigcup \oplus \hat{A_{\tau}}$, then $A=\bigcup \oplus A_{\tau}$ where $A=\hat{A} \cap L$ and $A_{\tau}=\hat{A}_{\tau} \cap L$.

Proof. Let $B=\bigcup \oplus A_{\tau}$. Then $B$ is a normal subspace of $L$ and $B \subset A$. Suppose $B \neq A$. Then, since $B$ and $A$ are both normal subspaces of $L, B$ is not dense in $A$. Let $B_{A}^{p}=B^{p} \cap A$. Then by [5, Theorem 29.10] $B^{p} \neq\{0\}$. Let $\hat{B}^{p}=\{b \in \hat{L}:|b| \leqq c$ for some $\left.c \in B^{p}\right\}$. Then, $\left\{\hat{B}_{A}^{p}\right\}$ is a normal subspace in $\hat{L}$, and $\left\{\hat{B}_{A}^{p}\right\} \neq\{0\}$. Moreover, $A_{\tau} \perp B_{A}^{p}$ for each $\tau$ and have $\hat{A}_{\tau} \perp \hat{B}_{A}^{p}$ for each $\tau$. But then since $\hat{A}_{\tau}$ is a normal subspace of $\hat{L}, \hat{A}_{\tau} \perp\left\{\hat{B}_{A}^{p}\right\}$ for each $\tau$. So, since $\left\{\hat{B}_{A}^{p}\right\} \neq\{0\}$, we have that $\hat{A} \cap\left\{\hat{B}_{A}^{p}\right\}^{p}$ is a normal subspace of $\hat{L}$ which contains $\hat{A_{\tau}}$ for each $\tau$ and yet, $\hat{A}$ contains it properly. This contradicts the hypothesis.

TheORem 2.15. Let $L$ be Archimedean. The carrier $L_{1}$ of $L_{n}^{\tilde{n}}$ is the Riesz direct sum of normal subspaces $A_{\tau}$ such that $A_{\tau}$ is super-Archimedean and has the Egoroff property, and in addition, each $A_{\tau}$ possesses a strictly positive normal integral.

Proof. By [4, Theorem 27.17], $\hat{L}_{1}=\bigcup \oplus \hat{A}_{\tau}$ where $\hat{A}_{\tau}$ is a normal subspace of $\hat{L}$ for each $\tau$. Then $L_{1}=\hat{L}_{1} \cap L=\bigcup \oplus A_{\tau}$ where $A_{\tau}=\hat{A_{\tau}} \cap L$ by 2.14. But $A_{\tau}$ is a normal subspace of $L$ and possesses the required properties by 2.13 .

The corollary to Theorem 27.17 in [4], can be generalized independently of the theorem.

THEOREM 2.16. If $L_{n}^{\tilde{n}}$ has a finite or countable order basis, then the carrier $L_{1}$ of $L_{n}^{\tilde{n}}$ is super-Archimedean.

Proof. By Corollary 27.18 in [4], $\hat{L}_{1}$ is super-Dedekind complete and hence by 5.1, $L_{1}$ is super-Archimedean, noting that $L_{1}=\hat{L}_{1} \cap L$.

Corollary 27.19 in [4] generalizes quite easily to Archimedean vector lattices, using 1.1:

THEOREM 2.17. (i) $L_{n}^{\tilde{n}}$ is the Riesz direct sum of normal subspaces $B_{\tau}$ such that each $B_{\tau}$ is super-Dedekind complete and has the Egoroff property.

(ii) If the carrier $L_{1}$ of $L_{n}^{\tilde{n}}$ has a finite or countable order basis, then $L_{\tilde{n}}^{\tilde{n}}$ is superDedekind complete.

Proof. (i) is an immediate consequence of 1.1. If $\hat{L}_{1}$ has a countable order basis 
then $L_{1}$ has a countable order basis since $L_{1}$ is order-dense in $L_{1}$. (ii) then follows from 1.1 and [4, Corollary 27.19].

Added in proof. In a subsequent paper, the authors of [4] give a proof of Theorem 1.1. They apply it to different problems.

\section{REFERENCES}

1. S. Kaplan, The second dual of the space of continuous functions. II, Trans. Amer. Math. Soc. 93 (1959), 329-350.

2. - The second dual of the space of continuous functions. III, Trans. Amer. Math. Soc. 101 (1961), 34-51.

3. - The second dual of the space of continuous functions. IV, Trans. Amer. Math. Soc. 113 (1964), 512-546.

4. W. A. J. Luxemburg and A. C. Zaanen, Notes on Banach function spaces. VIII, Nederl. Akad. Wetensch. Proc. Ser. A 67 (1964), 104-119.

5. - Notes on Banach function spaces. IX, Nederl. Akad. Wetensch. Proc. Ser. A 67 (1964), 360-376.

6. H. Nakano, Modern spectral theory, Maruzen, Tokyo, 1950.

Purdue University,

LAFAYETTE, INDIANA 